\title{
Autoestima, metas de logro y estrategias de autoprotección: Un enfoque centrado en la persona
}

\section{RESUMEN}

Las estrategias de self-handicapping y pesimismo defensivo constituyen un recurso frecuentemente utilizado por los estudiantes que buscan proteger sus sentimientos de valía personal. Numerosos trabajos han evidenciado que la autoestima y las metas de logro constituyen dos de los predictores motivacionales más importantes de estas estrategias. Sin embargo, el estudio de estas relaciones se ha efectuado desde un enfoque centrado en la variable. A partir de la identificación de diferentes perfiles de estudiantes en función de sus características de autoestima y metas de logro (aprendizaje, aproximación al rendimiento y evitación del rendimiento), se pretendía determinar, dentro de cada perfil, el poder predictivo de estas variables sobre las estrategias de autoprotección. En el estudio participaron 1028 estudiantes universitarios, seleccionados mediante un muestreo por conveniencia. Partiendo de la determinación de cuatro perfiles - (a) alta autoestima y predominio de aproximación al rendimiento; (b) alta autoestima y predominio de aprendizaje; (c) baja autoestima y predominio de metas de rendimiento; y (d) baja autoestima y altas metas generalizadas-, se realizaron análisis de regresión lineal (pasos sucesivos). Los resultados evidenciaron que, en los dos perfiles con alta autoestima, las metas de aprendizaje constituían un predictor negativo del self-handicapping. Asimismo, en los perfiles con baja autoestima, las metas de evitación del rendimiento (negativamente) y ambas metas de aproximación (positivamente) se mostraron como predictores del pesimismo defensivo. Estos resultados sugieren que, en aras de propiciar una prevención eficaz del uso de estrategias de autoprotección, es preciso conocer las características motivacionales de cada estudiante.
María del Mar Ferradás

Canedo ${ }^{i}$

Universidade da

Coruña, España

Carlos Freire Rodríguez ${ }^{i i}$ Universidade da Coruña, España

Bibiana Regueiro Fernández ${ }^{\text {iii }}$ Universidade de Santiago de Compostela, España

Tania Vieites Lestón ${ }^{\text {iv }}$ Universidade da Coruña, España

Carolina Rodríguez-

Llorente $^{v}$

Universidade da Coruña, España

Palabras-clave: Autoestima; Metas de logro; Perfiles motivacionales; Self-handicapping; Pesimismo defensivo 


\section{INTRODUCCIÓN}

En su teoría de la autovalía, Covington (1992) postula que la búsqueda de la autoaceptación constituye una prioridad para el ser humano. Esta necesidad impulsaría a las personas a proteger sus propias creencias de competencia personal, bien sea orientándose hacia la consecución de resultados exitosos o hacia la evitación de fracasos. Desde esta consideración, en un contexto altamente evaluativo como el académico, en el que la valía personal se asocia significativamente al rendimiento (Zheng et al., 2020), las expectativas de éxito y de fracaso (i.e., rendir por encima o por debajo de determinados estándares) regulan en alto grado las conductas de logro de los estudiantes (Muenks et al., 2018).

Para algunos aprendices, el fracaso puede suponer un mensaje emocionalmente tan devastador que la protección de los sentimientos de valía personal se convierte en su genuina prioridad académica, de tal forma que se involucran en la utilización de complejas estrategias anticipatorias con el propósito de desvincular un potencial fracaso de su presunta valía "real" (De Castella et al., 2013).

Dos de los mecanismos autoprotectores más prevalentes en el contexto académico son el self-handicapping y el pesimismo defensivo. El selfhandicapping constituye una estrategia de autosabotaje, en la medida en que el estudiante reduce sus propias posibilidades de éxito mediante la creación de algún impedimento que obstaculiza su desempeño académico (Török et al., 2018). Esta autoobstaculización puede desplegarse en forma de conductas manifiestas - self-handicapping conductual - como, por ejemplo, reducir el nivel de esfuerzo, procrastinar o sobreimplicarse hasta el agotamiento, o bien a través de meras formulaciones verbales — self-handicapping alegado -, tales como aducir ansiedad, cansancio o una enfermedad (Leary \& Shepperd, 1986). Con independencia de su tipología, el impedimento le permite al estudiante generar una convincente coartada ante un previsible fracaso, de modo que, a ojos de los demás, la competencia personal quedaría eximida de toda sospecha (Berglas \& Jones, 1978). El self-handicapping, así pues, resulta marcadamente eficaz en el corto plazo, por cuanto permite proteger los sentimientos de valía personal del estudiante (McCrea \& Hirt, 2001), pero sus supuestos beneficios se diluyen a la larga, dado que la utilización recurrente de la estrategia a menudo comporta importantes perjuicios académicos (Cano et al., 2018; Schwinger et al., 2014) y emocionales (Putwain, 2019; Zuckerman \& Tsai, 2005), especialmente acusados en el caso del selfhandicapping conductual (Xing et al., 2018).

Al igual que el self-handicapping, el pesimismo defensivo también se caracteriza por ser una estrategia de autoprotección de carácter anticipatorio. Consiste en el desarrollo de expectativas denodadamente bajas respecto al propio rendimiento, en virtud de las cuales el estudiante vislumbra con gran detalle el modo en que se producirá el esperado fracaso (Norem, 2008). Si bien estos negativos presagios resultan infundados a priori, habida cuenta de que, por lo común, al estudiante le precede una sólida trayectoria académica, la estrategia pesimista defensiva supone un mecanismo de protección frente a la ansiedad que genera la posibilidad de rendir por debajo de un determinado nivel. En efecto, mediante la reducción de las expectativas, el estudiante logra gestionar con eficacia su ansiedad (Seery et al., 2008) y comienza a planificar minuciosamente la forma en que puede evitar cada uno de los aspectos que ha anticipado le llevarán a fracasar 
(Chang \& Sivam, 2004). En la medida en que suele propiciar un buen rendimiento (Nob et al., 2018), el pesimismo defensivo resultaría mucho más adaptativo que el self-handicapping en términos académicos (Cano et al., 2018). Sin embargo, a largo plazo, la elevada ansiedad experimentada por los pesimistas defensivos acaba suponiendo un importante coste para su bienestar psicológico (Lee et al., 2010) e, incluso, para su rendimiento (Norem \& Cantor, 1990).

Pese a las consecuencias negativas reseñadas, la eficacia autoprotectora evidenciada por el self-handicapping y el pesimismo defensivo hace que resulte difícil disuadir a los estudiantes de su utilización (Norem, 2002; Snyder, 1984). Ello ha motivado una notable producción investigadora dirigida a conocer las variables motivacionales que incrementarían la vulnerabilidad del estudiante a ambas estrategias. Entre estas variables, la autoestima y las metas de logro destacan significativamente.

La autoestima constituye un juicio valorativo autorreferido que evidencia el grado en que la persona se aprecia y se siente una persona valiosa (Rosenberg, 1965). Si bien, por lo general, la autoestima es considerada un poderoso determinante de las conductas de logro de los estudiantes (Zheng et al., 2020), su vinculación con las estrategias de autoprotección de la valía ofrece ciertas inconsistencias. Así, se ha sugerido que tanto el self-handicapping como el pesimismo defensivo tienen su origen en autoesquemas negativos (García \& Pintrich, 1994; Norem, 2001), lo que vincularía a ambas estrategias con la baja autoestima (Ferradás et. al, 2019; Chen et al., 2018; Pullmann \& Allik, 2008; Yavuzer, 2015). Sin embargo, otros estudios han encontrado una relación entre la alta autoestima y las estrategias de self-handicapping y pesimismo defensivo (Kim et al., 2012; Rappo et al., 2017; Yamawaki et al., 2004). En este sentido, se ha aducido la posibilidad de que los estudiantes con alta autoestima recurran a estrategias autoprotectoras ya sea con el fin de preservar sus autovaloraciones en estándares elevados (Buckingham et al., 2019; Valle et al., 2005) o para engrandecerlas (Tice, 1991; Yamawaki et al., 2004).

Por su parte, las metas de logro representan los objetivos y propósitos de competencia que persiguen los estudiantes a la hora de implicarse en las tareas (Elliot et al., 2005). Generalmente, la investigación suele diferenciar entre metas de aprendizaje y metas de rendimiento (Elliot, 1999). Los estudiantes con metas de aprendizaje consideran la competencia personal como una característica maleable, que puede incrementarse por vía de la mejora personal y el dominio de las tareas (Martin et al., 2017). En consecuencia, se orientan hacia la consecución de estándares intrapersonales -i.e., satisfacer el interés y la curiosidad, mejorar las habilidades personales, dominar las tareas, etc.- (Hulleman et al., 2010). Por el contrario, los estudiantes que adoptan metas de rendimiento suelen concebir la competencia como un atributo inmodificable $\mathrm{y}$, como tal, algo que puede evidenciarse, pero no mejorarse (Burnette et al., 2013; Dweck, 2012). A tenor de esta creencia, algunos estudiantes se afanarán por lograr un rendimiento más alto que el de sus compañeros o bien por demostrar que poseen unas habilidades académicas superiores -i.e., metas de aproximación al rendimiento- - Sin embargo, otros estudiantes buscarán no rendir peor que sus compañeros o no obtener juicios sociales negativos respecto a su competencia académica — metas de evitación del rendimiento- (e.g., Elliot, 1999). 
En lo que respecta a la relación entre metas de logro y estrategias de autoprotección, por lo común, self-handicapping y pesimismo defensivo se relacionan con las metas de rendimiento.

En concreto, la investigación previa vincula el self-handicapping y el pesimismo defensivo con las metas de evitación del rendimiento (Akin, 2014; Chen et al., 2018; Schwinger \& Stiensmeier-Pelster, 2011), si bien parece que las metas de aproximación al rendimiento incrementarían también la tendencia a implicarse en estas dos estrategias autoprotectoras (Ferradás et al., 2019; Elliot \& Church, 2003; Lovejoy \& Durik, 2010). Por el contrario, no parece que bajo el self-handicapping y el pesimismo defensivo subyazca una orientación hacia metas de aprendizaje. En este sentido, algunos estudios muestran que las metas de aprendizaje se relacionan negativamente con el self-handicapping y el pesimismo defensivo (Elliot \& Church, 2003; Martin et al., 2001; Schwinger et al., 2014; Yamawaki et al., 2004). No obstante, otros trabajos han encontrado una orientación a metas de aprendizaje combinada con metas de rendimiento en estudiantes pesimistas defensivos (Ferradás et. al, 2018; Martin et al., 2003).

\section{EL PRESENTE ESTUDIO}

Si bien, de conformidad con la investigación previa, tanto la autoestima como las metas de logro parecen erigirse en factores motivacionales significativamente asociados a las estrategias de autoprotección, hasta la fecha, ambos factores han sido mayoritariamente analizados de forma aislada (i.e., independiente el uno del otro) en su relación con el self-handicapping y el pesimismo defensivo. Sin embargo, este enfoque centrado en la variable no lograría captar la compleja realidad motivacional presente en las aulas pues, como plantean Bråten y Olaussen (2005, p. 360), “... las personas se mueven en ambientes instruccionales, no a través de variables". En otras palabras, características motivacionales como la autoestima o las diferentes metas de logro no operarían de forma independiente, sino que se conjugarían de formas diferentes en cada estudiante, influyendo en sus conductas académicas. Analizar esta complejidad requiere, así pues, la adopción de un enfoque centrado en la persona (Abar \& Loken, 2010).

A diferencia de los enfoques centrados en la variable, el cual asume que todos los sujetos de una población son homogéneos respecto a un conjunto de parámetros (i.e., variables), los enfoques centrados en la persona consideran la posibilidad de que una población sea heterogénea en cuanto a la presencia de un conjunto de parámetros (Laursen \& Hoff, 2006). En consecuencia, los enfoques centrados en la persona pretenden determinar y describir un número óptimo de subpoblaciones, cada una de las cuales comparte ciertos atributos (i.e., poseen niveles similares respecto a un conjunto de variables), diferenciándose del resto de subgrupos (Collins \& Lanza, 2013). Estos enfoques, así pues, poseen un mayor grado de especificidad que los enfoques centrados en la persona, en la medida en que permiten identificar con mayor precisión la relación existente entre cada subpoblación y otras variables, de tal forma que los resultados pueden generalizarse a todos los sujetos representados por esa subpoblación (Howard \& Hoffman, 2018).

El enfoque centrado en la persona goza de una aceptable difusión en la investigación sobre metas de logro, dado que se ha evidenciado la existencia de perfiles de estudiantes que conjugan de diversas formas las distintas razones y propósitos que llevan al estudiante a implicarse en 
contextos de logro -i.e., perfiles de múltiples metas- (Ballard et al., 2018; Elliot \& Hulleman, 2017). Sin embargo, el trabajo de Ferradás et. al, 2019 constituye el único precedente que ha identificado la existencia de perfiles diferenciados de estudiantes en función de la forma en que en ellos se combinan la autoestima y las metas de logro (metas de aprendizaje, metas de aproximación al rendimiento y metas de evitación del rendimiento). En este estudio, se determinó la existencia de cuatro perfiles motivacionales de estudiantes universitarios: (a) alta autoestima, bajas metas de aprendizaje y altas metas de rendimiento (aproximativas y evasivas); (b) alta autoestima, altas metas de aprendizaje y bajas metas de rendimiento (aproximativas y evasivas); (c) baja autoestima, bajas metas de aprendizaje y altas metas de rendimiento (aproximativas y evasivas); y (d) baja autoestima, altas metas de aprendizaje, altas metas de aproximación al rendimiento y metas moderadas de evitación del rendimiento. Asimismo, se encontró que estos dos últimos perfiles mostraban niveles significativamente más elevados de selfhandicapping (conductual y alegado) y pesimismo defensivo, respectivamente.

El análisis de la relación entre los perfiles de autoestima y metas de logro y las estrategias de self-handicapping y pesimismo defensivo podría favorecer el diseño de intervenciones psicoeducativas más específicas $\mathrm{y}$, por ende, eficaces, para los estudiantes self-handicappers y pesimistas defensivos. Por ello, tomando como referencia los resultados de Ferradás et. al, 2019, el objetivo de la presente investigación es determinar, dentro de cada perfil motivacional, cuáles son las variables que se relacionan significativamente con las estrategias de autoprotección de la valía. Este estudio, por tanto, conjuga las aportaciones de los enfoques centrados en la persona - identificación de subgrupos homogéneos en cuanto al comportamiento de ciertas variables - y los enfoques centrados en la variable - una vez identificado cada subgrupo, el enfoque centrado en la variable permite explicar, en cada uno de ellos, el efecto de unas variables (i.e., autoestima y metas de logro) sobre otras (estrategias de autoprotección) con un mayor grado de parsimonia (Howard \& Hoffman, 2018).

\section{MÉTODO}

\subsection{PARTICIPANTES}

El estudio se realizó sobre una muestra de 1028 estudiantes (887 mujeres y 141 varones) de Ciencias de la Educación (Grados en Educación Infantil, Educación Primaria, Educación Social y Logopedia; $n=718$ ) y de Ciencias de la Salud (Grados en Fisioterapia, Enfermería y Podología; $n=310$ ) de la Universidade da Coruña (España), reclutados mediante un muestreo por conveniencia. Del total de participantes, 382 eran estudiantes de primer año, 334 , de segundo año y 312 , de tercero. El rango de edad de los participantes se situaba entre los 18 y los 25 años $\left(M_{\text {edad }}=21.36, D T_{\text {edad }}=3.81\right)$.

\subsection{INSTRUMENTOS}

Para la medida de la autoestima se utilizó la versión validada al español de la Rosenberg Self-esteem Scale (Martín-Albo et al., 2007). El instrumento incluye diez ítems (e.g., "En general, estoy satisfecho/a conmigo mismo/a), evaluados mediante una escala de respuesta Likert $(1=$ en total desacuerdo $-5=$ 
totalmente de acuerdo). En el presente estudio, la escala evidenció una adecuada consistencia interna: $\alpha=0.88 ; \omega=0.89$ (CI 95\% [.88, .90]).

Las metas de logro se evaluaron mediante la versión validada al contexto español de la Goal Orientation Scale (Jover et al., 2014). La subescala de metas de aprendizaje contiene seis ítems ("Para mí es importante aprender cosas nuevas en clase"), que evalúan el interés del estudiante por aprender y adquirir nuevos conocimientos. Su consistencia interna en nuestro estudio ha sido: $\alpha=0.79, \omega=0.79$ (CI 95\% [.77, .81]). La subescala de metas de aproximación al rendimiento $(\alpha=0.85, \omega=0.85$ (CI 95\% $[.83, .86])$ comprende cinco ítems (e.g., "Intento sacar mejores notas que mis compañeros/as") que analizan en qué medida el estudiante tiene un afán por demostrar una superioridad competencia sobre sus compañeros. Finalmente, la subescala de evitación del rendimiento (seis ítems; e.g., "Cuando en clase respondo de forma incorrecta, me preocupa lo que mis compañeros/as puedan pensar de mí"; $\alpha=0.80, \omega=0.80$ (CI 95\% [.78, .82]) evalúa la motivación del estudiante por evitar juicios sociales negativos. Se utilizó una escala de respuesta Likert entre 1 = nunca y 5 = siempre.

Para evaluar el self-handicapping se utilizó la adaptación española de la Self-Handicapping Scale (Ferradás et. al, 2018). El instrumento mide dos tipologías de self-handicapping: self-handicapping conductual, que evalúa conductas manifiestas (nueve ítems; e.g., "Tiendo a no intentar hacer las tareas, así tengo una excusa si no lo hago tan bien como esperaba"; $\alpha=0.84$; $\omega=0.84$ (CI 95\% [.82, .85]); y self-handicapping alegado, que mide hándicaps verbalizados (16 ítems; e.g., "Les digo a los demás que estoy más agotado de lo que realmente estoy cuando tengo que hacer tareas o exámenes, así que, si no lo hago tan bien como esperaba, puedo decir que esa es la razón"; $\alpha=0.90$; $\omega=0.91$ (CI 95\% $[.90, .91])$.

Por su parte, la medida del pesimismo defensivo se efectuó mediante la adaptación española del Defensive Pessimism Questionnaire (Norem, 2002). El instrumento consta de 12 ítems (e.g., "Considerar lo que puede ir mal me ayuda a prepararme"), que ha evidenciado una consistencia interna adecuada en nuestro estudio: $\alpha=0.89 ; \omega=0.89$ (CI 95\% [.88, .90]). En ambos instrumentos sobre estrategias de autoprotección se utilizó una escala de respuesta Likert de cinco puntos ( $1=$ nunca $-5=$ siempre $)$.

\subsection{PROCEDIMIENTO}

La investigación fue llevada a cabo de conformidad con los protocolos éticos de la Universidade da Coruña y la Declaración de Helsinki. A los participantes se les informó acerca de los objetivos del estudio y se les solicitó su colaboración voluntaria y desinteresada. Asimismo, se les indicó que su identidad permanecería en el anonimato y sus datos serían plenamente confidenciales. Una vez informados de las características del estudio, se les solicitó su consentimiento informado por escrito respecto a la participación en el estudio. La recogida de datos se realizó en las aulas en las que los estudiantes recibían su formación universitaria de forma habitual, dentro del horario académico y en una única sesión sin límite de tiempo. Los cuestionarios fueron aplicados de forma individual por personal investigador entrenado. Con el fin de reducir el efecto del orden de presentación de los instrumentos, se efectuó un contrabalanceo completo. Para ello, las cuatro escalas utilizadas se presentaron aleatoriamente en posiciones diferentes del cuestionario, atendiendo a seis combinaciones. Asimismo, a los participantes 
se les proporcionó la dirección de correo de la investigadora principal con objeto de que, en caso de estar interesados, pudiesen informarse sobre los resultados del estudio.

\subsection{ANÁLISIS DE DATOS}

Se tomaron como referencia los cuatro perfiles motivacionales identificados por Ferradás et. al, 2019 mediante un análisis de perfiles latentes (LPA). En dicho trabajo, se determinó, comparativamente con los modelos de dos, tres, cinco y seis grupos, la idoneidad de la solución de cuatro grupos, sobre la base de los indicadores aportados por los siguientes parámetros estadísticos: AIC (criterio de información de Akaike), BIC (criterio de información bayesiana de Schwarz) y SSA-BIC (BIC ajustado por el tamaño de la muestra), prueba formal de la razón de máxima verosimilitud ajustada de Lo, Mendell y Rubin (LMRT), prueba paramétrica de la razón de verosimilitud de reemplazamiento (PBLRT), ausencia de clases espurias (grupos conformados por menos del 5\% del total de la muestra), y precisión clasificatoria del modelo de acuerdo con los coeficientes de probabilidades a posteriori y el estadístico de entropía (para una caracterización completa del proceso de identificación de los perfiles, véase Ferradás et. al, 2019).

En el presente estudio, en cada uno de los cuatro perfiles, se realizó un análisis de regresión lineal. Habida cuenta de la ausencia de un marco conceptual y empírico consistente respecto al papel desempeñado por la autoestima y las diferentes metas de logro (metas de aprendizaje, metas de aproximación al rendimiento y metas de evitación del rendimiento) en su relación con las estrategias de self-handicapping y pesimismo defensivo, resulta plausible considerar que alguna o algunas de las variables independientes pudieran resultar irrelevantes en la explicación de los modelos de regresión. En consecuencia, se optó por el método por pasos sucesivos, en virtud del cual, en cada perfil motivacional analizado, se selecciona, a cada paso, la variable independiente que (a) supera el criterio de entrada (criterio de significación estadística basado en la probabilidad de F) y (b) más alto correlaciona con la variable dependiente. Asimismo, cada vez que se introduce una variable al modelo, las variables previamente incluidas son nuevamente evaluadas, de tal forma que, si no cumplen los criterios de salida (probabilidad estadística de $F$ ), son eliminadas del modelo. Todos los análisis estadísticos se realizaron con el software estadístico SPSS 26.

\section{RESULTADOS}

VARIABLES MOTIVACIONALES SIGNIFICATIVAMENTE RELACIONADAS CON LAS ESTRATEGIAS DE AUTOPROTECCIÓN EN EL PERFIL ALTA AUTOESTIMA, BAJAS METAS DE APRENDIZAJE Y ALTAS METAS DE RENDIMIENTO (APROXIMATIVAS Y EVASIVAS)

En la Tabla 1 se recogen los resultados del análisis de regresión realizado respecto a los predictores motivacionales (autoestima y metas de logro) significativos de las estrategias de autoprotección en el perfil alta autoestima, bajas metas de aprendizaje y altas metas de rendimiento (aproximativas y evasivas). De acuerdo con estos resultados, en este perfil de estudiantes, las metas de aprendizaje se erigieron en un predictor negativo significativo del self-handicapping conductual $(\beta=-0.20, p<0.05)$. Por su parte, las metas de evitación del rendimiento predijeron positiva y significativamente el self- 
handicapping alegado $(\beta=0.22, p<0.05)$. Ninguna de las variables motivacionales evidenció una capacidad predictiva significativa sobre el pesimismo defensivo.

Tabla 1

Variables motivacionales significativamente relacionadas con las estrategias de autoprotección en el perfil alta autoestima, bajas metas de aprendizaje y altas metas de rendimiento (aproximativas y evasivas)

\begin{tabular}{|c|c|c|c|c|c|c|c|}
\hline $\begin{array}{l}\text { Perfil AAE/BMA/AMAR/AMER } \\
(n=153)\end{array}$ & \multicolumn{2}{|c|}{ Autoestima } & \multicolumn{2}{|c|}{ MA } & \multicolumn{2}{|c|}{ MAR } & \multirow{2}{*}{$\begin{array}{c}\text { MER } \\
3.98 \\
(0.45)\end{array}$} \\
\hline$M(D T)$ & \multicolumn{2}{|c|}{$3.99(0.16)$} & \multicolumn{2}{|c|}{$2.21(0.64)$} & \multicolumn{2}{|c|}{$3.94(0.32)$} & \\
\hline Variables motivacionales & VD & $\mathbf{R}^{2}$ & B & EE & $\boldsymbol{\beta}$ & $F$ & $p$ \\
\hline Aprendizaje & $\mathrm{SHC}$ & .038 & -.175 & .07 & -.196 & 6.008 & .015 \\
\hline Evitación del rendimiento & SHA & .050 & .330 & .12 & .224 & 7.986 & .005 \\
\hline
\end{tabular}

Nota. AAE/BMA/AMAR/AMER = Alta Autoestima/Bajas Metas de Aprendizaje/Altas Metas de Aproximación al Rendimiento/Altas Metas de Evitación del Rendimiento; $M A=$ Metas de Aprendizaje; $M A R=$ Metas de Aproximación al Rendimiento; MER = Metas de Evitación del Rendimiento; SHC = Self-handicapping Conductual; SHA = Selfhandicapping Alegado.

VARIABLES MOTIVACIONALES SIGNIFICATIVAMENTE RELACIONADAS CON LAS ESTRATEGIAS DE AUTOPROTECCIÓN EN EL PERFIL ALTA AUTOESTIMA, ALTAS METAS DE APRENDIZAJE Y BAJAS METAS DE RENDIMIENTO (APROXIMATIVAS Y EVASIVAS)

Respecto al perfil alta autoestima, altas metas de aprendizaje y bajas metas de rendimiento (aproximativas y evasivas), tal y como se muestra en la Tabla 2 , las metas de aprendizaje también se mostraron como un predictor significativo negativo del self-handicapping conductual $(\beta=-0.11, p<.05)$. Esta misma orientación a metas evidenció ser un predictor negativo significativo $(\beta=-0.13, p<0.001)$ del self-handicapping alegado. La utilización de esta estrategia autoprotectora se encontró también significativamente explicada (positivamente) por las metas de evitación del rendimiento $(\beta=0.12, p<0.001)$. En este perfil, ninguno de los factores motivacionales analizados se erigió en predictor significativo del pesimismo defensivo.

Tabla 2 .

Variables motivacionales significativamente relacionadas con las estrategias de autoprotección en el perfil alta autoestima, altas metas de aprendizaje y bajas metas de rendimiento (aproximativas y evasivas)

\begin{tabular}{|c|c|c|c|c|c|c|c|}
\hline $\begin{array}{l}\text { Perfil AAE/AMA/BMAR/BMER } \\
\qquad(n=520)\end{array}$ & \multirow{2}{*}{\multicolumn{2}{|c|}{$\begin{array}{l}\text { Autoestima } \\
3.71(0.16)\end{array}$}} & \multirow{2}{*}{\multicolumn{2}{|c|}{$\begin{array}{c}\text { MA } \\
3.54(0.60)\end{array}$}} & \multirow{2}{*}{$\begin{array}{c}\text { MAR } \\
2.59(0.70)\end{array}$} & \multirow{2}{*}{\multicolumn{2}{|c|}{$\begin{array}{c}\text { MER } \\
2.78(0.86)\end{array}$}} \\
\hline$M(\mathrm{DT})$ & & & & & & & \\
\hline Variables motivacionales & VD & $\mathbf{R}^{2}$ & B & EE & $\boldsymbol{\beta}$ & $F$ & $p$ \\
\hline Aprendizaje & $\mathrm{SHC}$ & .012 & -.116 & .05 & -.108 & 6.169 & .013 \\
\hline $\begin{array}{c}\text { Aprendizaje } \\
\text { Evitación del rendimiento }\end{array}$ & SHA & .032 & $\begin{array}{l}-.136 \\
.087\end{array}$ & $\begin{array}{l}.04 \\
.03\end{array}$ & $\begin{array}{l}-.132 \\
.120\end{array}$ & 8.606 & $<.001$ \\
\hline
\end{tabular}

Nota. AAE/AMA/BMAR/BMER = Alta Autoestima/Altas Metas de Aprendizaje/Bajas Metas de Aproximación al Rendimiento/Bajas Metas de Evitación del Rendimiento; MA = Metas de Aprendizaje; MAR = Metas de Aproximación al Rendimiento; MER = Metas de Evitación del Rendimiento; SHC = Self-handicapping Conductual; SHA = Selfhandicapping Alegado. 
VARIABLES MOTIVACIONALES SIGNIFICATIVAMENTE RELACIONADAS CON LAS ESTRATEGIAS DE AUTOPROTECCIÓN EN EL PERFIL BAJA AUTOESTIMA, BAJAS METAS DE APRENDIZAJE Y ALTAS METAS DE RENDIMIENTO (APROXIMATIVAS Y EVASIVAS)

En el perfil baja autoestima, bajas metas de aprendizaje y altas metas de rendimiento (aproximativas y evasivas), únicamente las metas de evitación del rendimiento se mostraron como un predictor significativo de las estrategias de autoprotección (véase Tabla 3). En concreto, esta orientación a metas se erigió en un predictor significativo negativo del pesimismo defensivo $(\beta=-0.22, p<0.05)$. Sin embargo, en este perfil de estudiantes, ninguna de las variables motivacionales analizadas mostró una capacidad explicativa significativa sobre las estrategias de self-handicapping.

Tabla 3

Variables motivacionales significativamente relacionadas con las estrategias de autoprotección en el perfil baja autoestima, bajas metas de aprendizaje y altas metas de rendimiento (aproximativas y evasivas)

\begin{tabular}{ccccccccc}
\hline $\begin{array}{c}\text { Perfil BAE/BMA/AMAR/AMER } \\
(\boldsymbol{n}=\mathbf{1 2 4})\end{array}$ & \multicolumn{2}{c}{ Autoestima } & \multicolumn{2}{c}{ MA } & \multicolumn{2}{c}{ MAR } & MER \\
\hline $\boldsymbol{M}(\mathbf{D T})$ & $2.75(0.90)$ & 1.62 & $(0.34)$ & $3.96(0.34)$ & $4.17(0.31)$ \\
\hline Variables motivacionales & VD & $\mathbf{R}^{2}$ & $\mathbf{B}$ & $\mathrm{EE}$ & $\boldsymbol{\beta}$ & $\boldsymbol{F}$ & $\boldsymbol{p}$ \\
\hline Evitación del rendimiento & PD & .050 & -.744 & .29 & -.224 & 6.439 & .012 \\
\hline
\end{tabular}

Nota. BAE/BMA/AMAR/AMER = Baja Autoestima/Bajas Metas de Aprendizaje/Altas Metas de Aproximación al

Rendimiento/Altas Metas de Evitación del Rendimiento; $M A=$ Metas de Aprendizaje; $M A R=$ Metas de Aproximación al Rendimiento; $M E R=$ Metas de Evitación del Rendimiento; PD = Pesimismo Defensivo.

VARIABLES MOTIVACIONALES SIGNIFICATIVAMENTE RELACIONADAS CON LAS ESTRATEGIAS DE AUTOPROTECCIÓN EN EL PERFIL BAJA AUTOESTIMA, ALTAS METAS DE APRENDIZAJE, ALTAS METAS DE APROXIMACIÓN AL RENDIMIENTO Y METAS MODERADAS DE EVITACIÓN DEL RENDIMIENTO

Finalmente, en la Tabla 4 se presentan los resultados del análisis de regresión relativos al perfil baja autoestima, altas metas de aprendizaje, altas metas de aproximación al rendimiento y metas moderadas de evitación del rendimiento. Las metas de aprendizaje se mostraron como un predictor significativo del self-handicapping alegado y del pesimismo defensivo. En el primer caso, de índole negativa $(\beta=-0.16, p<0.05)$ y en el segundo, positiva $(\beta=0.14, p<0.001)$. Asimismo, respecto al pesimismo defensivo, se encontró que esta estrategia está explicada positiva y significativamente por las metas de aproximación al aprendizaje $(\beta=0.53, p<0.001)$ en los estudiantes encuadrados en este perfil. Sin embargo, ninguna de las variables motivacionales consideradas se mostró como predictora significativa del selfhandicapping conductual.

Tabla 4

Variables motivacionales significativamente relacionadas con las estrategias de autoprotección en el perfil BAE/AMA/AMAR/MMER

\begin{tabular}{|c|c|c|c|c|c|c|c|}
\hline $\begin{array}{l}\text { Perfil BAE/AMA/AMAR/MMER } \\
\qquad(n=231)\end{array}$ & \multirow{2}{*}{\multicolumn{2}{|c|}{$\begin{array}{l}\text { Autoestima } \\
2.74(0.99)\end{array}$}} & \multirow{2}{*}{\multicolumn{2}{|c|}{$\begin{array}{c}\text { MA } \\
4.15(0.39)\end{array}$}} & \multirow{2}{*}{\multicolumn{2}{|c|}{$\begin{array}{c}\text { MAR } \\
4.10(0.53)\end{array}$}} & \multirow{3}{*}{$\begin{array}{c}\text { MER } \\
\frac{3.33(0.48)}{p}\end{array}$} \\
\hline \multirow{2}{*}{$\begin{array}{c}M(\mathrm{DT}) \\
\text { Variables motivacionales }\end{array}$} & & & & & & & \\
\hline & VD & $\mathbf{R}^{2}$ & B & $\mathrm{EE}$ & $\boldsymbol{\beta}$ & $F$ & \\
\hline \multirow{3}{*}{$\begin{array}{c}\text { Aprendizaje } \\
\text { Aproximación al rendimiento } \\
\text { Aprendizaje } \\
\end{array}$} & SHA & .026 & -.237 & .09 & -.162 & 6.199 & .013 \\
\hline & \multirow{2}{*}{ PD } & \multirow{2}{*}{.329} & .617 & .07 & .526 & 55.97 & \multirow{2}{*}{$<.001$} \\
\hline & & & .229 & .09 & .143 & 1 & \\
\hline
\end{tabular}

Nota. BAE/AMA/AMAR/MMER = Baja Autoestima/Altas Metas de Aprendizaje/Altas Metas de Aproximación al Rendimiento/Moderadas Metas de Evitación del Rendimiento; MA = Metas de Aprendizaje; $M A R=$ Metas de Aproximación al Rendimiento; MER = Metas de Evitación del Rendimiento; SHA = Self-handicapping Alegado; PD = Pesimismo Defensivo. 


\section{DISCUSIÓN}

La adopción de un enfoque centrado en la persona goza de una atención creciente en el estudio de la motivación académica. Si bien este interés es especialmente palpable en un tópico como el de las metas de logro, con la proliferación de trabajos basados en la perspectiva de las múltiples metas, el estudio sobre cómo el estudiante conjuga estas razones y propósitos de logro con otras variables motivacionales es todavía precario. Hasta la fecha, el trabajo de Ferradás et. al, 2019 constituye el único precedente que ha determinado la existencia de perfiles diferenciados de estudiantes en virtud de su autoestima y sus metas de logro (metas de aprendizaje, metas de aproximación al rendimiento y metas de evitación del rendimiento). Tomando como referencia los cuatro perfiles identificados en ese estudio, el presente trabajo pretendía ahondar en la comprensión de la relación entre los perfiles motivacionales y las estrategias de autoprotección de la valía personal. En concreto, se analizó, dentro de cada perfil, qué variables motivacionales se relacionaban significativamente con la tendencia de los estudiantes universitarios a implicarse o inhibirse en la adopción de mecanismos de self-handicapping y pesimismo defensivo.

De acuerdo con nuestros resultados, los cuatro perfiles muestran algunas diferencias en su relación con las estrategias de autoprotección. De este modo, se observó que, en los dos perfiles con alta autoestima - perfil alta autoestima, bajas metas de aprendizaje y altas metas de rendimiento (aproximativas y evasivas); y perfil alta autoestima, altas metas de aprendizaje y bajas metas de rendimiento (aproximativas y evasivas)-, las metas de aprendizaje y las metas de evitación del rendimiento serían los principales factores motivacionales que se relacionarían con las estrategias de self-handicapping conductual y alegado.

En concreto, en el perfil alta autoestima, bajas metas de aprendizaje $\mathrm{y}$ altas metas de rendimiento (aproximativas y evasivas), las metas de aprendizaje evidenciaron una relación negativa con el self-handicapping conductual, mientras que las metas de evitación del rendimiento mostraron una relación positiva con el self-handicapping alegado. Estos hallazgos parecen indicar que nos encontramos ante un perfil de estudiantes que, aunque se quieren y valoran a sí mismos, están especialmente preocupados por su imagen de competencia (tanto en lo que respecta a demostrar su superioridad respecto a los compañeros como a evitar juicios sociales negativos), de tal forma que basarían su percepción de valía personal en su rendimiento. Por el contrario, su interés por aprender y mejorar académicamente sería escaso. En la medida en que estas características se asocian a estudiantes que poseen una concepción inmodificable de la competencia personal (Burnette et al., 2013; Dweck, 2012), a tenor de nuestros resultados, el escaso afán por acrecentar sus habilidades académicas se relacionaría, en estos estudiantes, con una mayor tendencia a enrolarse en conductas activas de self-handicapping (e.g., procrastinar, reducir el esfuerzo) como mecanismo de autoprotección ante un previsible fracaso. Por su parte, la elevada motivación por evitar la crítica negativa se encontraría asociada al afán por verbalizar la existencia de impedimentos como coartada ante un previsible bajo rendimiento.

Por lo que respecta al perfil alta autoestima, altas metas de aprendizaje y bajas metas de rendimiento (aproximativas y evasivas), de forma similar al perfil anterior, las metas de aprendizaje se mostraron 
negativamente relacionadas con el self-handicapping (en este caso, de ambos tipos de autoobstaculización), y las metas de evitación del rendimiento, positivamente con el self-handicapping alegado. Se trata, así pues, de un perfil de estudiantes que muestran actitudes positivas hacia sí mismos y se enfocan hacia su propia mejora como estudiantes, mientras que su preocupación respecto a lo que otras personas puedan pensar de sus cualidades académicas sería reducida. Estas características motivacionales, fundamentalmente vinculadas a un interés genuino por aprender $\mathrm{y}$ perfeccionarse académicamente, así como a una escasa preocupación por que un potencial bajo rendimiento conlleve juicios sociales negativos respecto a su propia competencia, se relacionarían con una menor probabilidad de acogerse a mecanismos de self-handicapping. Desde esta consideración, el perfil alta autoestima, altas metas de aprendizaje y bajas metas de rendimiento (aproximativas y evasivas) resultaría motivacionalmente más adaptativo que el perfil alta autoestima, bajas metas de aprendizaje y altas metas de rendimiento (aproximativas y evasivas).

En conjunto, los resultados relativos a estos dos perfiles son consistentes con la tesis de que el self-handicapping constituye una estrategia eminentemente autopresentacional (Rhodewalt, 2008) y, por ende, vinculada a la necesidad de protección de la imagen social de competencia personal. Asimismo, a tenor de nuestros hallazgos, parece que esta necesidad de proteger la propia imagen mediante mecanismos de autoobstaculización estaría especialmente ligada a estudiantes con alta autoestima, tal y como han concluido otros trabajos mediante un enfoque centrado en la variable (Kim et al., 2012; Rappo et al., 2017). Por tanto, es posible que los estudiantes encuadrados en el perfil alta autoestima, bajas metas de aprendizaje y altas metas de rendimiento (aproximativas y evasivas) busquen, mediante el selfhandicapping, preservar su autoestima en altos niveles (Buckingham et al., 2019) o, incluso, engrandecerla (Tice, 1991). En este sentido, los datos del presente estudio indican que la querencia por el self-handicapping, evidenciada por los estudiantes encuadrados en este perfil, vendría motivacionalmente explicada por la adopción de metas de evitación del rendimiento. Ello corroboraría los hallazgos de otros estudios que han encontrado una relación positiva entre esta orientación a metas y el selfhandicapping (Akin, 2014; Ntoumanis et al., 2009).

Ahora bien, nuestros hallazgos posibilitarían profundizar en la comprensión de esta relación pues, por un lado, permiten caracterizar con mayor precisión el perfil motivacional de los estudiantes con altas metas de evitación del rendimiento que se implican en el self-handicapping (i.e., alta autoestima, altas metas de aproximación al rendimiento, bajas metas de aprendizaje). Por otro lado, nuestros resultados parecen indicar que el afán por evitar una imagen social de incompetencia se vincularía en mayor medida a la adopción del self-handicapping de índole alegada. Finalmente, otra interesante lectura que puede extraerse del análisis relativo a los perfiles alta autoestima, bajas metas de aprendizaje y altas metas de rendimiento (aproximativas y evasivas) y alta autoestima, altas metas de aprendizaje y bajas metas de rendimiento (aproximativas y evasivas) anida en el posible papel protector de las metas de aprendizaje frente al selfhandicapping, en la línea sugerida por otros estudios (e.g., Schwinger \& Stiensmeier-Pelster, 2011).

Por lo que se refiere a los dos perfiles con baja autoestima - perfil baja autoestima, bajas metas de aprendizaje y altas metas de rendimiento 
(aproximativas y evasivas); y perfil baja autoestima, altas metas de aprendizaje, altas metas de aproximación al rendimiento y metas moderadas de evitación del rendimiento-, se evidenció en ellos una relación más importante con el pesimismo defensivo. En concreto, en el perfil baja autoestima, bajas metas de aprendizaje y altas metas de rendimiento (aproximativas y evasivas), las metas de evitación del rendimiento se mostraron negativamente relacionadas con esta estrategia autoprotectora. Por tanto, si bien desde un enfoque centrado en la variable, la investigación previa vincula la utilización del pesimismo defensivo con las metas de evitación al rendimiento (e.g., Chen et al., 2018; Yamawaki et al., 2004), nuestros datos no avalan esta relación o, cuando menos, no en estudiantes que conjugan esta orientación a metas con otros propósitos de logro (altas metas de aproximación al rendimiento y bajas metas de aprendizaje), así como una baja autoestima. De hecho, las metas de evitación del rendimiento tampoco parecen relacionarse significativamente con la utilización del pesimismo defensivo en los estudiantes que encarnan el perfil baja autoestima, altas metas de aprendizaje, altas metas de aproximación al rendimiento y metas moderadas de evitación del rendimiento. Esta aparente contradicción con los resultados de otros estudios podría tener que ver con el hecho de que, en el presente trabajo, las metas de evitación del rendimiento han sido definidas bajo un criterio normativo (evitar juicios sociales negativos). En este sentido, se ha señalado que en los estudiantes pesimistas defensivos no existiría un afán autopresentacional (Norem, 2002), de tal forma que la preocupación por un bajo rendimiento tendría que ver más con una razón de autoprotección que de evitar críticas negativas de otras personas respecto a la competencia (Norem \& Illingworth, 1993).

Finalmente, el perfil de estudiantes con baja autoestima, altas metas de aprendizaje, altas metas de aproximación al rendimiento y metas moderadas de evitación del rendimiento es el que parece más proclive a implicarse en el pesimismo defensivo. Así, de conformidad con los resultados obtenidos, tanto las metas de aprendizaje como las metas de aproximación al rendimiento se mostraron positivamente relacionados con esta estrategia. Por tanto, nos encontramos ante un perfil de estudiantes que presentan juicios autorreferidos negativos respecto a la valía personal, con un moderado temor al bajo rendimiento, pero que también muestran un elevado afán por aprender y obtener altas calificaciones académicas, siendo estos dos últimos factores los que se relacionarían en mayor medida con la utilización del pesimismo defensivo.

Estos datos vendrían a refrendar la dualidad motivacional que parece subyacer en los pesimistas defensivos, en virtud de la cual estarían cognitivamente comprometidos con el miedo al fracaso, pero conductualmente orientados al éxito (Martin \& Marsh, 2003). En este sentido, algunos trabajos han enfatizado que en el pesimismo defensivo existe un interés por la automejora (Norem \& Smith, 2006), aunque dicho interés podría constituir, para los estudiantes que utilizan esta estrategia, un medio para lograr altos estándares de rendimiento (Martin et al., 2003). No sorprende, por ello, que el pesimismo defensivo se relacione con la utilización eficaz de estrategias de autorregulación para el aprendizaje y, en general, con un buen desempeño académico (Cano et al., 2018; Elliot \& Church, 2003; Nob et al., 2018).

Además de la vinculación con el pesimismo defensivo, es preciso destacar que, en los estudiantes encuadrados en el perfil baja autoestima, 
altas metas de aprendizaje, altas metas de aproximación al rendimiento y metas moderadas de evitación del rendimiento, las altas metas de aprendizaje se mostraron negativamente asociadas al self-handicapping alegado. Este dato, así pues, vendría a corroborar la importante relación existente entre estas metas de logro y el self-handicapping, tal y como se evidenció también en el perfil alta autoestima, altas metas de aprendizaje y bajas metas de rendimiento (aproximativas y evasivas).

\section{IMPLICACIONES EDUCATIVAS}

Los resultados del presente estudio pueden conllevar algunas implicaciones educativas de consideración. De esta forma, la identificación de las variables que, dentro de cada perfil motivacional, tendrían una relación significativa con la utilización del self-handicapping y del pesimismo defensivo puede contribuir a determinar qué factores deben priorizarse de cara a prevenir o reducir la implicación de los estudiantes universitarios en estos mecanismos autoprotectores en función de sus características de autoestima y metas de logro. Asumiendo que tanto el self-handicapping como el pesimismo defensivo se asocian con importantes perjuicios personales y académicos a largo plazo (Lee et al., 2010; Norem \& Cantor, 1990; Putwain, 2019; Schwinger et al., 2014; Xing et al., 2018), nuestros datos sugieren que las metas de aprendizaje, con independencia del nivel de autoestima del estudiante, podrían suponer un importante factor de protección frente al selfhandicapping. Por el contrario, el énfasis en el rendimiento situaría el foco en la comparación social (e.g., evitar la crítica), lo que se relacionaría con una tendencia más elevada a involucrarse en mecanismos de autoobstaculización, especialmente en estudiantes con alta autoestima. Por tanto, en aras de reducir la utilización del self-handicapping, es preciso promover contextos de aprendizaje que enfaticen el valor del aprendizaje y la mejora basada en estándares intrapersonales (Urdan \& Midgley, 2003).

En el caso del pesimismo defensivo, nuestros hallazgos indican que esta estrategia se encuentra motivacionalmente vinculada a metas de aprendizaje y metas de aproximación al rendimiento. Si bien, en general, las metas de aprendizaje suelen ser caracterizadas como altamente adaptativas (Nadon et al., 2020), los beneficios asociados a las metas de aproximación al rendimiento resultan más controvertidos, especialmente en lo que respecta a la vertiente emocional (Senko \& Dawson, 2017). En este sentido, a tenor de los resultados del presente estudio, es preciso tomar en consideración que, aunque asociado a metas de índole aproximativa, el pesimismo defensivo es más proclive en estudiantes con baja autoestima y moderado temor al fracaso. Por tanto, dentro de este perfil motivacional parece tan importante estimular su afán por aprender cómo reducir su preocupación por el rendimiento. Desde esta consideración, convendría eliminar en estos estudiantes la asociación entre rendimiento y competencia personal (Martin, 2010). Ello se vería facilitado si, como apuntan Thompson \& Parker (2007), se promueven ambientes de aprendizaje que favorecen la percepción de control en el estudiante (e.g., explicitar y consensuar criterios de evaluación, promover evaluaciones formativas, alentar a los estudiantes a atribuir sus resultados al empleo adecuado o inadecuado de estrategias). 


\section{LIMITACIONES DEL ESTUDIO}

Estas aportaciones deben ser analizadas con cautela, habida cuenta de las limitaciones que presenta el estudio realizado. En primer lugar, la naturaleza transversal del diseño de investigación no permite establecer relaciones de causalidad entre las variables analizadas. Así pues, a tenor de nuestros hallazgos, no es posible determinar si las variables motivacionales analizadas (autoestima y metas de logro) constituyen antecedentes o consecuentes de las estrategias de autoprotección o, incluso, evidenciar la posible existencia de una relación recíproca entre estos constructos. En segundo lugar, el procedimiento de muestreo llevado a cabo carece del rigor suficiente como para posibilitar la generalización de los resultados obtenidos al conjunto de la población universitaria. En tercer lugar, la recogida de datos basada exclusivamente en procedimientos de autoinforme podría suponer un sesgo a la hora de obtener información válida y fiable. Finalmente, en este estudio no se han contemplado otras variables (e.g., metas sociales, metas de evitación del trabajo, variables de personalidad) que podrían tener un peso significativo en la explicación del self-handicapping y del pesimismo defensivo.

\section{AGRADECIMIENTOS}

Los autores agradecen a los participantes su colaboración desinteresada en el estudio.

\section{REFERENCIAS}

Abar, B., \& Loken, E. (2010). Self-regulated learning and self-directed study in a pre-college sample. Learning and Individual Differences, 20(1), 25 29. https://doi.org/10.1016/j.lindif.2009.09.002

Akin, U. (2014). 2x2 achievement goal orientations and selfhandicapping. Ceskoslovenska Psichologie, 58(5), 431-441.

Ferradás, M. M., Freire, C., Regueiro, B., \& Valle, A. (2018). Defensive pessimism, self-esteem and achievement goals: A person-centered approach. Psicothema, 30(1), 53-58. https://doi.org/10.7334/psicothema2017.199

Ferradás, M. M., Freire, C., Núñez, J. C., \& Regueiro, B. (2019). Associations between profiles of self-esteem and achievement goals and the protection of selfworth in university students. International Journal of Environmental Research and Public Health, 16(12), 2218. https://doi.org/10.3390/ijerph16122218

Ballard, T., Vancouver, J. B., \& Neal, A. (2018). On the pursuit of multiple goals with different deadlines. Journal of Applied Psychology, 103(11), 1242-1264. https://doi.org/10.1037/apl0000304

Berglas, S., \& Jones, E. (1978). Drug choice as a self-handicapping strategy in response to noncontingent success. Journal of Personality and Social Psychology, 36(4), 405-417. https://doi.org/10.1037/0022-3514.36.4.405

Bråten, I., \& Olaussen, B. S. (2005). Profiling individual differences in student motivation: A longitudinal cluster-analytic study in different academic contexts. Contemporary Educational Psychology, 30(3), 359-396.

https://doi.org/10.1016/j.cedpsych.2005.01.003

Buckingham, J. T., Lam, T. A, Andrade, F. C, Boring, B. L., \& Emery, D. (2019). Reducing contingent self-worth: A defensive response to self-threats. The 
Journal of Social Psychology, 159(3), 284-298.

https://doi.org/10.1080/00224545.2018.1461604

Burnette, J. L., O'Boyle, E. H., VanEpps, E. M., Pollack, J. M., \& Finkel, E. J. (2013). Mind-sets matter: A meta-analytic review of implicit theories and selfregulation. Psychological Bulletin, 139(3), 655-701.

https://doi.org/10.1037/a0029531

Cano, F., Martin, A. J., Ginns, P., \& Berbén, A. B. G. (2018). Students' selfworth protection and approaches to learning in higher education: predictors and consequences. Higher Education, 76, 163-181. https://doi.org/10.1007/s10734-0170215-0

Chang, W. C., \& Sivam, R. W. (2004). Constant vigilance: Heritage values and defensive pessimism in coping with severe acute respiratory syndrome in Singapore. Asian Journal of Social Psychology, 7(1), 35-53. https://doi.org/10.1111/j.1467-839x.2004.00133.x

Chen, Z., Sun, K., \& Wang, K. (2018). Self-Esteem, achievement goals, and self-handicapping in college physical education. Psychological Reports, 121(4), 690704. https://doi.org/10.1177/0033294117735333

Collins, L. M., \& Lanza, S. T. (2013). Latent class and latent transition analysis: With applications in the social, behavioral, and health sciences. John Wiley.

Covington, M. V. (1992). Making the grade: A self-worth perspective on motivation and school reform. Cambridge University Press.

De Castella, K., Byrne, D., \& Covington, M. V. (2013). Unmotivated or motivated to fail? A cross-cultural study of achievement motivation, fear of failure, and student disengagement. Journal of Educational Psychology, 105(3), 861-880. https://doi.org/10.1037/a0032464

Dweck, C. S. (2012). Mindset: How you can fulfil your potential. Constable \& Robinson Limited.

Elliot, A. J. (1999). Approach and avoidance motivation and achievement goals. Educational Psychologist, 34(3), 169-189. http://dx.doi.org/10.1207/s15326985ep3403_3

Elliot, A. J., \& Church, M. (2003). A motivational analysis of defensive pessimism and self-handicapping. Journal of Personality, 71(3), 369-396.

http://dx.doi.org/10.1111/1467-6494.7103005

Elliot, A. J., \& Hulleman, C. S. (2017). Achievement goals. In A. J. Elliot, C. S. Dweck, \& D. S. Yeager (Eds.), Handbook of competence and motivation: Theory and application (pp. 43-60). The Guilford Press.

Elliot, A. J., Shell, M. V., Henry, K. B., \& Maier, M. A. (2005). Achievement goals, performance contingencies, and performance attainment: An experimental test. Journal of Educational Psychology, 97(4), 630-640.

https://doi.org/10.1037/0022-0663.97.4.630

García, T., \& Pintrich, P. R. (1994). Regulating motivation and cognition in the classroom: The role of self-schemas and self-regulatory strategies. In D. H. Schunk \& B. J. Zimmerman (Eds.), Self-regulation of learning and performance. Issues and educational applications (pp. 127-156). Lawrence Erlbaum.

Howard, M. C., \& Hoffman, M. E. (2018). Variable-centered, personcentered, and person-specific approaches: Where theory meets the method. Organizational Research Methods, 21(4), 846-876.

https://doi.org/10.1177/1094428117744021

Hulleman, C. S., Schrager, S. M., Bodman, S. M., \& Harackiewicz, J. M. (2010). A me-ta-analytic review of achievement goal measures: Different labels for the same constructs or different constructs with similar labels? Psychollogical Bulletin, 136(3), 422-449. https://doi.org/10.1037/a0018947 
Jover, I., Navas, L., \& Holgado, F. P. (2014). Goal orientations in the students of the Education Faculty of Alicante. International Journal of Developmental and Educational Psychology, 1(2), 575-584.

Kim, H., Lee, K., \& Hong, Y. (2012). Claiming the validity of negative ingroup stereotypes when foreseeing a challenge: A self-handicapping account. Self and Identity, 11(3), 285-303. https://doi.org/10.1080/15298868.2011.561560

Laursen, B., \& Hoff, E. (2006). Person-centered and variable-centered approaches to longitudinal data. Merrill-Palmer Quarterly, 52(3), 377-389. https://doi.org/10.1353/mpq.2006.0029

Leary, M. R., \& Shepperd, J. A. (1986). Behavioral self-handicapping vs. self-reported handicaps: A conceptual note. Journal of Personality and Social Psychology, 51(6), 1265-1268. https://doi.org/10.1037/0022-3514.51.6.1265

Lee, J.-Z., Chen, C.-Y., \& Liang, T.-L. (2010). A motivational analysis of defensive pessimist and long-term wellbeing after achievement feedback. Bulletin of Educational Psychology, 41(4), 733-749.

Lovejoy, C. M., \& Durik, A. M. (2010). Self-handicapping: The interplay between self-set and assigned achievement goals. Motivation and Emotion, 34(3), 242-252. https://doi.org/10.1007/s11031-010-9179-4

Martin, A. J. (2010). Building classroom success: Eliminating academic fear and failure. Bloomsbury Publishing.

Martin, A. J., Bostwick, K., Collie, R. J., \& Tarbetsky, A. (2017). Implicit theories of intelligence. In V. Zeigler-Hill \& T.K. Shackelford (Eds.), Encyclopedia of Personality and Individual Differences (pp. 1-7). Springer. https://doi.org/10.1007/9783-319-28099-8_980-1

Martin, A. J., \& Marsh, H. W. (2003) Fear of failure: friend or foe? Australian Psychologist, 38(1), 31-38. https://doi.org/10.1080/00050060310001706997

Martin, A. J., Marsh, H. W., \& Debus, R. L. (2001). Self-handicapping and defensive pessimism: Exploring a model of predictors and outcomes from a selfprotection perspective. Journal of Educational Psychology, 93(1), 87-102.

https://doi.org/10.1037/0022-0663.93.1.87

Martin, A. J., Marsh, H. W., Williamson, A., \& Debus, R. L. (2003). Selfhandicapping, defensive pessimism and goal orientation: A qualitative study of university students. Journal of Educational. Psychology, 95(3), 617-628.

https://doi.org/10.1037/0022-0663.95.3.617

Martín-Albo, J., Núñez, J. L., Navarro, J. G., \& Grijalvo, F. (2007). The Rosenberg Self-Esteem Scale: Translation and validation in university students. Spanish Journal of Psychology, 10(2), 458-467. https://doi.org/10.1017/S1138741600006727

McCrea, S. M., \& Hirt, E. R. (2001). The role of ability judgments in selfhandicapping. Personality and Social Psychology Bulletin, 27(10), 1378-1389. https://doi.org/10.1177/01461672012710013

Muenks, K., Wigfield, A., \& Eccles, J. S. (2018). I can do this! The development and calibration of children's expectations for success and competence beliefs. Developmental Review, 48, 24-39. https://doi.org/10.1016/j.dr.2018.04.001

Nadon, L., Babenko, O., Chazan, D., \& Daniels, L. M. (2020). Burning out before they start? An achievement goal theory perspective on medical and education students. Social Psychology of Education, 23, 1055-1071.

https://doi.org/10.1007/s11218-020-09572-0

Nob, R. M., Bumanglag, A. M. L., Diwa, G. M., \& Ponce, G. I. (2018). The moderating role of defensive pessimism in the relationship between test anxiety and performance in a licensure examination. Educational Measurement and Evaluation Review, 9(1), 68-83. 
Norem, J. K. (2001). Defensive pessimism, optimism, and pessimism. In E. C. Chang (Ed.), Optimism and pessimism: Implications for theory, research and practice (pp. 77-100). American Psychological Association.

Norem, J. K. (2002). El poder positivo del pensamiento negativo: utiliza el pesimismo defensivo para reducir tu ansiedad y rendir al máximo [The positive power of negative thinking: Using defensive pessimism to harness anxiety and perform at your peak]. Paidos.

Norem, J. K. (2008). Defensive pessimism as a self-critical tool. In E. C. Chang (Ed.), Self-criticism and self-enhancement: Theory, research and clinical applications (pp. 89-104). American Psychological Association.

Norem, J. K., \& Cantor, N. (1990). Cognitive strategies, coping, and perceptions of competence. In R. J. Sternberg \& J. Kolligian jr. (Eds.), Competence considered (pp. 190-204). Yale University Press.

Norem, J. K., \& Illingworth, K. S. S. (1993). Strategy dependent effects of reflecting on self and tasks: Some implications of optimism and defensive pessimism. Journal of Personality and Social Psychology, 65(4), 822-835.

http://dx.doi.org/10.1037/0022-3514.65.4.822

Norem, J. K., \& Smith, S. (2006). Defensive pessimism: Positive past, anxious present, and pessimistic future. In L. J. Sanna y E. C. Chang (Eds.), Judgments over time: The interplay of thoughts, feelings, and behaviors (pp. 34-46). University Press.

Ntoumanis, N., Thogersen-Ntoumani, C., \& Smith, A. (2009). Achievement goals, self-handicapping and performance: A $2 \times 2$ achievement goal perspective. Journal of Sports Sciences, 27, 1471-1482. https://doi.org/10.1080/02640410903150459

Pullmann, H., \& Allik, J. (2008). Relations of academic and general selfesteem to school achievement. Personality and Individual Differences, 45(6), 559-564. https://doi.org/10.1016/j.paid.2008.06.017

Putwain, D. W. (2019). An examination of the self-referent executive processing model of test anxiety: control, emotional regulation, self-handicapping, and examination performance. European Journal of Psychology of Education, 34, 341358. https://doi.org/10.1007/s10212-018-0383-z

Rappo, G., Alesi, M., \& Pepi, A. (2017). The effects of school anxiety on self-esteem and self-handicapping in pupils attending primary school. European Journal of Developmental Psychology, 14(4), 465-476.

https://doi.org/10.1080/17405629.2016.1239578

Rhodewalt, F. (2008). Self-Handicapping: On the self-perpetuating nature of defensive behavior. Social and Personality Psychology Compass, 2(3), 1255-1268. https://doi.org/10.1111/j.1751-9004.2008.00117.x

Rosenberg, M. (1965). Society and the adolescent self-image. Princeton University.

Schwinger, M., \& Stiensmeier-Pelster, J. (2011). Prevention of selfhandicapping - The protective function of mastery goals. Learning and Individual Differences, 21(6), 699-709. https://doi.org/10.1016/j.lindif.2011.09.004

Schwinger, M., Wirthwein, L., Lemmer, G., \& Steinmayr, R. (2014). Academic self-handicapping and achievement: A meta-analysis. Journal of Educational Psychology, 106(3), 744-761. https://doi.org/10.1037/a0035832

Seery, M., West, T., Weisbuch, M., \& Blascovich, J. (2008). The effects of negative reflection for defensive: Dissipation or harnessing of threat? Personality and Individual Differences, 45(6), 515-520. https://doi.org/10.1016/j.paid.2008.06.004

Senko, C., \& Dawson, B. (2017). Performance-approach goal effects depend on how they are defined: Meta-analytic evidence from multiple educational 
outcomes. Journal of Educational Psychology, 109(4), 574-

598. https://doi.org/10.1037/edu0000160

Snyder, C. R. (1984). Excuses, excuses: They sometimes actually work to relieve the burden of blame. Psychology Today, 18, 50-55.

Thompson, T., \& Parker, C. (2007). Diagnosing the poor performance of self-worth protective students: A product of failure outcome uncertainty, evaluative threat, or both? Educational Psychology, 27(1), 111-134.

http://dx.doi.org/10.1080/01443410601061512

Tice, D. M. (1991). Esteem protection or enhancement? Self-handicapping motives and attributions differ by trait self-esteem. Journal of Personality and Social Psychology, 60(5), 711-725. http://doi.org/10.1037/0022-3514.60.5.711

Török, L., Szabó, Z. P., \& Tóth, L. (2018). A critical review of the literature on academic self-handicapping: Theory, manifestations, prevention and measurement. Social Psychology of Education: An International Journal, 21(5), 11751202. https://doi.org/10.1007/s11218-018-9460-z

Urdan, T., \& Midgley, C. (2003). Changes in the perceived clasroom goal structure and pattern of adaptative learning during early adolescence. Contemporary Educational Psychology, 28(4), 524-551. https://doi.org/10.1016/S0361476X(02)00060-7

Valle, A., Cabanach, R. G., Rodríguez, S., Núñez, J. C., \& González-Pienda, J. A. (2005). Self-worth protection strategies in higher educational students: Exploring a model of predictors and consequences. In R. Nata (Ed.), New directions in higher education (pp. 99-126). Nova Science Publishers.

Xing, S., Gao, X., Jiang, Y., Archer, M., \& Liu, X. (2018). Effects of ability and effort praise on children's failure attribution, self-handicapping, and performance. Frontiers in Psychology, 9, 1883. https://doi.org/10.3389/fpsyg.2018.01883

Yamawaki, N., Tschanz, B. T., \& Feick, D. L. (2004). Defensive pessimism, self-esteem instability and goal strivings. Cognition and Emotion, 18(2), 233-249. http://doi.org/10.1080/02699930341000004

Yavuzer, Y. (2015). Investigating the relationship between selfhandicapping tendencies, self-esteem and cognitive distortions. Educational Sciences: Theory \& Practice, 15(4), 879-890. https://doi.org/10.12738/estp.2015.4.2434

Zheng, L. R., Atherton, O. E., Trzesniewski, K., \& Robins, R. W. (2020). Are self-esteem and academic achievement reciprocally related? Findings from a longitudinal study of Mexican-origin youth. Journal of Personality, 88(6), 1058-1074. https://doi.org/10.1111/jopy.12550

Zuckerman, M., \& Tsai, F. F. (2005). Costs of self-handicapping. Journal of Personality, 73(2), 411-442. https://doi.org/10.1111/j.1467-6494.2005.00314.x 
Informação dos autores

i Universidade da Coruña, España.

https://orcid.org/0000-0002-9716-8306

ii Universidade da Coruña, España.

https: //orcid.org/0000-0002-6252-4016

iii Universidade de Santiago de Compostela, España. https://orcid.org/0000-0001-8519-960X

iv Universidade da Coruña, España.

https://orcid.org/0000-0003-4440-0201

v Universidade da Coruña, España.

https: //orcid.org/0000-0002-2894-5271

Toda a correspondência relativa a este artigo deve ser enviada para:

Carlos Freire Rodríguez

carlos.freire.rodriguezaudc.es

Recebido em 8 de setembro de 2020

Aceite para publicação em 23 de junho de 2021 
Self-esteem, achievement goals, and self-protection strategies: A person-centred approach

\begin{abstract}
Self-handicapping and defensive pessimism strategies are a resource frequently used by students seeking to protect their feelings of personal worth. A large group of studies has shown that self-esteem and achievement goals are two of the most important motivational predictors of these strategies. However, the study of these relationships has been carried out from a variable-centred approach. From the identification of different student profiles based on their self-esteem and achievement goals (learning, performance-approach, and performance-avoidance), it was intended to determine, within each profile, the predictive power of these variables on the self-protection strategies. 1,028 university students participated in the study, selected through convenience sampling. Starting from the determination of four profiles -(a) high self-esteem and predominance of performance goals; (b) high self-esteem and predominance of learning goals; (c) low self-esteem and predominance of performance goals; and (d) low self-esteem and high generalized goals-, linear regression analyses (stepwise method) were performed. The results showed that learning goals were a negative predictor of self-handicapping in the two profiles with high self-esteem. Likewise, performance-avoidance goals (negatively) and both approximative goals (positively) were shown as predictors of defensive pessimism in the profiles with low self-esteem. These results suggest that it is necessary to know the motivational characteristics of each student in order to promote effective prevention of the use of self-protection strategies.
\end{abstract}

Keywords: Self-esteem; Achievement goals; Motivational profiles; Self-handicapping; Defensive pessimism 


\section{Autoestima, metas de realização e estratégias de autoproteção: Uma abordagem centrada na pessoa}

\section{RESUMO}

Self-handicapping e pessimismo defensivo são estratégias frequentemente usadas por alunos que buscam proteger seus sentimentos de valor pessoal. Numerosos estudos demonstraram que a autoestima e as metas de realização são dois dos mais importantes preditores motivacionais dessas estratégias. No entanto, o estudo dessas relações foi realizado a partir de uma abordagem centrada na variável. A partir da identificação de diferentes perfis de alunos com base em suas características de autoestima e metas de realização (aprendizagem, ego-aproximação e ego-evasão), pretendeu-se determinar, dentro de cada perfil, o poder preditivo dessas variáveis sobre o uso de estratégias de autoproteção. Participaram do estudo 1.028 universitários, selecionados por amostragem por conveniência. Partindo da determinação de quatro perfis -(a) autoestima elevada e predomínio de metas ego; (b) autoestima elevada e predomínio de metas de aprendizagem; (c) baixa autoestima e predomínio de metas ego; e (d) baixa autoestima e metas generalizadas altas - foram realizadas análises de regressão linear (método stepwise). Os resultados mostraram que, nos dois perfis com elevada autoestima, as metas de aprendizagem foram um preditor negativo de self-handicapping. Da mesma forma, nos perfis com baixa autoestima, as metas ego-evasão (negativamente) e ambas as metas de aproximação (positivamente) mostraram-se preditores de pessimismo defensivo. Esses resultados sugerem que, para promover uma prevenção efetiva do uso de estratégias de autoproteção, é necessário conhecer as características motivacionais de cada aluno.

Palavras-chave: Autoestima; Metas de realização; Perfis motivacionais; Self-handicapping;

Pessimismo defensivo 